avances

en ciencias e

ingenierías

II Simposio
Latinoamericano de

Aplicaciones

Nucleares

en la

Agricultura

\title{
Tratamiento postcosecha con radiación gamma para extender la vida útil de papa chaucha amarilla (Solanum phureja)
}

\author{
Eddy Paul Anguisaca Totasig', María Cristina Cuesta Plúa', Marco Vinicio Sinche Serra” ${ }^{1 *}$
}

'Departamento de Ciencias Nucleares, Facultad de Ingeniería Química y Agroindustria, Escuela Politécnica Nacional, Quito, Ecuador

*Autor para correspondencia / Corresponding author, e-mail: marco.sinche@epn.edu.ec

\section{Post-harvest treatment with gamma radiation to increase shelf life of yellow chaucha potato Solamun phureja}

\section{Resumen}

Solanum phureja es una variedad andina de papa con un alto potencial de exportación, gracias a sus propiedades nutricionales, organolépticas y funcionales; sin embargo, su tiempo de vida en percha es muy reducido. Esta investigación tuvo como objetivo evaluar el tratamiento de este tubérculo con radiación gamma para retardar la brotación. Se empleó un diseño factorial $4 \times 2$ para seleccionar la mejor combinación de dosis $(0$, 80, 120 o 160 Gy) y tiempo después de la cosecha (uno o cinco días) del tratamiento. Asimismo, se utilizó un diseño factorial $3 \times 2$ para seleccionar la mejor combinación de dosis de clorprofam (CIPC) (0, $\left.20 \circ 30 \mathrm{mg} \mathrm{kg}^{-1}\right)$ y tiempo después de la cosecha (uno o cinco días); este es un tratamiento químico común para retrasar la germinación de la papa. El mejor tratamiento físico correspondió a la irradiación con una dosis de 120 Gy, cinco días después de la cosecha; que produjo una pérdida de peso de 16.5\%, una pérdida de firmeza de $17.2 \%$, ausencia de brotes y $4.0 \%$ de pudrición, después de 40 días de almacenamiento a $19^{\circ} \mathrm{C}$ y $60 \%$ de humedad relativa. El mejor tratamiento químico correspondió a la aplicación de $30 \mathrm{mg}$ de CIPC kg-1 a los cinco días de la cosecha, y produjo una pérdida de peso del 17.4\%, una pérdida de firmeza del $21.0 \%$, un $8.0 \%$ de podredumbre y la aparición de brotes después de 25 días. En las papas de la muestra control (sin ningún tratamiento), la pérdida de peso fue del 23.7\%, la pérdida de firmeza del 20.9\%, la pudrición del $7.0 \%$ y la aparición de brotes a los seis días. Los análisis sensoriales (apariencia, color, textura y sabor) y proximal (contenido de humedad, cenizas, proteína, extracto etéreo, fibra cruda y carbohidratos totales) revelaron que los tratamientos aplicados no afectaron las características organolépticas y nutricionales de las papas. Estos resultados sugieren que la irradiación de chaucha amarilla permite incrementar su vida útil en aproximadamente 33 días, conservando su calidad.

Palabras clave: Clorprofam, Pudrición, Radiaciones ionizantes, Tubérculo.

Editado por /

Edited by:

Gabriela Albán

Recibido /

Received:

05/10/2020

Aceptado /

Accepted:

24/10/2020

Publicado en línea /

Published online:

01/04/2021 


\begin{abstract}
Solanum phureja is an andean potato variety with a high export potential, thanks to its nutritional, organoleptic and functional properties; however, its shelf life is very short. The aim of this research was to evaluate the treatment of this tuber with gamma radiation to delay sprouting. A $4 \times 2$ factorial design was used to select the best dose $(0,80,120$ or 160 Gy) and time after harvest (one or five days) of the treatment. Similarly, a $3 \times 2$ factorial design was used to select the best chlorpropham (CIPC) dose $\left(0,20\right.$ or $\left.30 \mathrm{mg} \mathrm{kg}^{-1}\right)$ and time after harvest (one or five days). This is a common chemical treatment to delay sprouting in potato. The best physical treatment corresponded to the irradiation with a dose of 120 Gy, five days after harvest; it produced a weight loss of $16.5 \%$, a firmness loss of $17.2 \%$, no sprouts and $4.0 \%$ of rottenness after 40 days of storage at $19^{\circ} \mathrm{C}$ and $60 \%$ relative humidity. The best chemical treatment corresponded to the application of $30 \mathrm{mg} \mathrm{CIPC} \mathrm{kg}^{-1}$ five days after harvest, and it produced a weight loss of $17.4 \%$, a firmness loss of $21.0 \%, 8.0 \%$ of rottenness and the appearance of sprouts after 25 days. In the potatoes of the control sample (without any treatment), weight loss was $23.7 \%$, firmness loss was $20.9 \%$, rottenness of $7.0 \%$ and the appearance of sprouts after six days. Sensory analysis (appearance, color, texture and flavor) and proximal analysis (moisture, ashes, protein, lipids, crude fiber and carbohydrates content) showed that the applied treatments did not significantly affect the organoleptic or nutritional content of the potatoes. These results suggest that irradiation of Solanum phureja could increase its shelf life and keep the quality by approximately 33 days.
\end{abstract}

Keywords: Chlorpropham, lonizing radiations, Rottenness, Tuber.

\title{
INTRODUCCIÓN
}

La papa Solanum tuberosum se originó y domesticó en los Andes. Es el cuarto producto agrícola de mayor consumo a nivel mundial, luego del trigo, maíz y arroz [1]. Por su alto consumo y distribución global, fue definida con el lema "Papa, alimento ayer, hoy y siempre" en el XXVI Congreso de la Asociación Latinoamericana de la Papa-ALAP en el 2014 [2]. Un 50\% de la producción mundial de papa se consume en fresco, el resto se destina a la elaboración de productos e ingredientes alimenticios procesados, piensos de animales, semillas, industria farmacéutica y de combustibles [1]. En Ecuador, el porcentaje de papa que se destina al consumo en fresco es mayor al promedio mundial, aunque se ha observado una tendencia a la baja, de un 90\% en 2002 a un 74\% en 2012 $[3,4]$. En los Andes de Perú, Bolivia y Ecuador crecen más de 4000 variedades nativas, entre ellas se encuentra la papa chaucha amarilla Solanum phureja [1]. Esta variedad se caracteriza por una corta vida postcosecha, debido a la aparición de brotes entre cinco y ocho días luego de la cosecha $[5,6]$, o inclusive antes $[7,8]$, pero tiene un corto ciclo vegetativo (110 a 120 días) que permite establecer de tres a cuatro ciclos de siembracosecha por año $[9,10]$.

Por su corta vida útil, la comercialización de chaucha amarilla se torna difícil y los productores sufren altas pérdidas económicas. No se reportan datos en Ecuador, pero en el vecino país de Colombia la variedad chaucha amarilla registra pérdidas 
postcosecha de hasta un 50\% en el almacenamiento, transporte e industrialización [11]. Por lo tanto, la inhibición de brotes durante el periodo de dormancia de los tubérculos es de gran importancia económica. Para ello, se utiliza el almacenamiento refrigerado a temperatura entre 4 y $10{ }^{\circ} \mathrm{C}$ y humedad relativa (HR) de 85 a 90\%, y la aplicación de reguladores de crecimiento; en países tropicales se utiliza la combinación de ambos [12].

Por más de 50 años, el isopropil 3-clorocarbanilato [13, 14], clorprofam, CIPC o N-3clorofenil isopropil carbamato ha sido utilizado como el principal inhibidor de brotes en papas, pero en la actualidad existen preocupaciones por el alto grado de toxicidad que exhiben los metabolitos presentes o formados en los tubérculos tratados con él [12]. Por su extensivo uso, además, se presenta el riesgo de contaminación de los tubérculos que se usan como semilla, lo que provoca un retraso en el crecimiento de las plantas y una baja producción de tubérculos [15]. La Organización de Protección de Plantas de Norte América (NAPPO por sus siglas en inglés) establece como límite máximo de residuos de CIPC en papas frescas para consumo humano el valor de $15 \mathrm{mg} \mathrm{kg}^{-1}$ para Canadá y de $30 \mathrm{mg} \mathrm{kg}^{-1}$ para Estados Unidos [12]. Con el fin de asegurar la seguridad alimentaria y satisfacer la demanda de los consumidores por alimentos naturales, frescos, con mejor calidad nutricional y con una vida útil amplia, existe la necesidad de aplicar nuevas tecnologías o de modificar o combinar las técnicas tradicionales de conservación [16].

Paul Ezekiel y Pandey [12] discutieron la necesidad de buscar alternativas más seguras y efectivas que el CIPC y señalaron al tratamiento con radiación gamma como una opción. El Organismo Internacional de Energía Atómica (IAEA, por sus siglas en inglés) manifiesta que la irradiación de alimentos constituye una tecnología multipropósito y comprende un proceso mediante el cual un producto alimenticio se expone a radiaciones ionizantes con el fin de mantener su calidad e inocuidad [17]. La radiación ionizante actúa en tres fases: (1) acción física primaria sobre los átomos, la cual provoca la formación de moléculas ionizadas y radicales libres; (2) cambios químicos, que forman compuestos radioquímicos estables, de constitución determinada por la composición y estructura molecular del material tratado; (3) efectos biológicos, que incluyen la modificación en la membrana celular, reducción de la actividad enzimática y, por consecuencia, la reducción de la síntesis de ácidos nucleicos, lo cual afecta el metabolismo energético de la fosforilación y altera la composición del ADN [18].

La irradiación se utiliza a escala comercial para esterilizar, favorecer el control de plagas, prevenir enfermedades transmitidas por alimentos y retardar la germinación y maduración de frutas y vegetales [19]. Cada año se incrementan a nivel mundial los centros multipropósito de irradiación y, por ende, el número de alimentos procesados y productos agrícolas tratados principalmente con irradiación gamma [20,21]. El IAEA ha propuesto dosis entre 0.1 y 1.0 kGy para la inhibición de brotes y el retraso en la maduración de bulbos y tubérculos [17].

En 1958, la ex Unión Soviética autorizó el consumo de papas irradiadas, desde entonces se han llevado a cabo numerosos estudios acerca de la aplicación de radiación gamma sobre este producto [22]. La irradiación es una técnica postcosecha eficaz para el almacenamiento de papas en condiciones no refrigeradas; se han reportado también valores de 0.08 y $0.12 \mathrm{kGy}$ como las mejores dosis para mantener las propiedades físicas y mecánicas en la variedad "Kufri Sindhuri", almacenada a $22{ }^{\circ} \mathrm{C}$ durante 120 días [23]. 
Otros estudios en la variedad "Ágata", almacenada por 35 días a $21{ }^{\circ} \mathrm{C}$ y $39 \%$ de HR, reportaron $0.15 \mathrm{kGy}$ como la mejor dosis para reducir pérdidas postcosecha [24]. Para el caso de la variedad chaucha amarilla, Zavala [25] evaluó los efectos de diferentes dosis de radiación gamma $(0.025 ; 0.050 ; 0.075$ y 0.10 kGy) sobre la expresión fenotípica; la dosis de 0.050 kGy indujo el retraso en la brotación y existieron cambios morfológicos en la coloración y forma de hojas y flores. Guzman, [26] realizó la caracterización fenotípica empleando radiación gamma sobre papa chaucha amarilla y determinó que no se inhibe el proceso de germinación, solamente se retrasa el tiempo de emergencia de los brotes, al aplicar una dosis relativamente baja de 0.025 kGy.

El objetivo de esta investigación fue estudiar el efecto del tratamiento con radiación gamma sobre la vida útil de la papa chaucha amarilla en condiciones de almacenamiento no refrigerado $\left(19^{\circ} \mathrm{C}\right.$ y $60 \%$ de humedad relativa) en la ciudad de Quito y compararlo con la aplicación de CIPC, como tratamiento referencial.

\section{MATERIALES Y MÉTODOS}

\section{Materiales y equipos}

Se utilizó como materia prima tubérculos de chaucha amarilla cosechados el 16 y 20 de julio de 2017 (Grado A; 10\% con no más del 1\% de pudriciones húmeda y parda, de tercera de acuerdo con su tamaño de entre 30 y $44 \mathrm{~mm}$ de diámetro) en la parroquia Toacazo, cantón Latacunga, provincia de Cotopaxi. Se consideraron los requisitos de calidad para consumo humano, establecidos en la Norma NTN INEN 1516 [27].

Los reactivos utilizados fueron clorprofam o CIPC (Sigma Aldrich; pureza > 98\%) y alcohol industrial (WEIR; pureza > 96\%) para diluir el CIPC.

Los equipos utilizados fueron un irradiador panorámico con una fuente de cobalto 60, una balanza digital BODECO BPS-51 y un penetrómetro (MCCORMICK FT-11; con sonda plana de $6 \mathrm{~mm}$ de diámetro).

\section{Selección de la mejor dosis de irradiación}

Las papas cosechadas fueron lavadas con agua potable para eliminar residuos de tierra y se secaron a condiciones ambientales de la parroquia Toacazo $\left(15^{\circ} \mathrm{C}\right.$ y $60 \%$ de humedad relativa (HR)). Luego, se realizó la clasificación, selección, empacado y etiquetado en saquillos de nylon de $1 \mathrm{~kg}$ cada uno. Las muestras se transportaron a la ciudad de Quito y se almacenaron a condiciones ambientales (en promedio $19^{\circ} \mathrm{C}$ y $60 \%$ de HR), a fin de emular la forma en la que comúnmente se almacenan las papas en esta ciudad. Los tubérculos cosechados el 16 (después de un día o 1D) y el 20 de julio de 2017 (después de cinco días o 5D), fueron irradiados el 21 de julio de 2017, con la fuente de cobalto 60 ubicada en el Laboratorio de Tecnología de Radiaciones (LTR) que pertenece a la Escuela Politécnica Nacional (EPN). Los saquillos fueron ubicados a $0.3 \mathrm{~m}$ del contorno de la fuente y los tiempos de irradiación fueron de 56, 83 y 111 min, en función de la dosis requerida. 
Se utilizó un diseño experimental $4 \times 2$, en el que las variables de diseño fueron la dosis de radiación gamma $(0,80,120$ y 160 Gy) y el número de días desde la cosecha hasta la irradiación (1D y 5D). Se realizaron tres repeticiones; las unidades experimentales fueron saquillos de $1 \mathrm{~kg}$ de tubérculos. En el producto tratado se evaluaron parámetros de calidad y se hizo un análisis proximal y un análisis sensorial, según se describe posteriormente.

\section{Selección de la mejor dosis de CIPC}

Las papas lavadas y seleccionadas fueron tratadas con clorprofam. Se utilizó un diseño experimental $3 \times 2$, en el que las variables de diseño fueron la dosis de $\operatorname{CIPC}(0,20$ y 30 mg de CIPC $\mathrm{kg}^{-1}$ de papa) y el número de días desde la cosecha hasta la irradiación (1D y 5 D); se realizaron tres repeticiones.

Para ambos diseños experimentales, las muestras control correspondieron a papas del mismo tiempo postcosecha sin ningún tratamiento.

\section{Evaluación de los parámetros de calidad poscosecha}

Las muestras irradiadas y tratadas con CIPC fueron almacenadas en un cuarto oscuro a condiciones ambientales. Los parámetros de calidad postcosecha: brotación externa, diámetro y longitud de los brotes, pérdida de peso, pudrición, pérdida de firmeza, apariencia general y tiempo de vida útil, fueron evaluados los lunes, miércoles y viernes durante 40 días luego de la irradiación o la aplicación de CIPC.

\section{Brotación externa}

Se determinó la brotación externa mediante el contaje del número de tubérculos con brotes visibles [28] y se reportó su valor como porcentaje, con respecto al número total (10) de tubérculos examinados por cada repetición [29]; es decir que, una brotación externa del $0 \%$ significaría que ninguna de las papas evaluadas presentó brotes visibles.

\section{Diámetro y longitud de los brotes}

La medición del diámetro y de la longitud de los brotes se realizó con ayuda de una regla de acetato; se registraron los valores de un total de 10 tubérculos examinados por cada repetición.

\section{Pérdida de peso}

Los saquillos con papas fueron pesados antes de la aplicación de los tratamientos y cada dos días durante el periodo de almacenamiento, en una balanza electrónica BODECO BPS51 (4000 g $\pm 0.1 \mathrm{~g}$ ). Los resultados se reportaron como porcentaje de peso perdido [24].

\section{Pudrición}

La pudrición fue determinada mediante el pesaje de los tubérculos afectados con síntomas de descomposición o desintegración de tejidos. El valor obtenido se reportó como porcentaje con respecto al peso total del saquillo de cada tratamiento [29]. 


\section{Pérdida de firmeza}

Para la valoración de la pérdida de firmeza, se midió este parámetro cada viernes, durante los 40 días de almacenamiento, con un penetrómetro McCORMICK FT-11 de sonda plana de $6 \mathrm{~mm}$ de diámetro; se realizaron tres lecturas en la zona ecuatorial de los tubérculos por cada tratamiento [24].

\section{Apariencia general y tiempo de vida útil}

Para valorar la apariencia general de las papas, se tomó en cuenta el encogimiento, la marchitez y daños físicos en la superficie de los tubérculos. Se utilizó una escala de uno a cinco, según los siguientes criterios: (de 4 a 5) los tubérculos mantienen las características iniciales, (de 3 a 4) los tubérculos presentan cierto deterioro y daño, pero aún son aptos para comercialización, (de 1 a 3) los tubérculos no son comercializables [30]. Para la valoración del tiempo de vida útil se marcó el día en el cual los brotes alcanzaron 2 mm de longitud, de acuerdo con la Norma NTN INEN 1516:2012 [27] y también se tomó en cuenta la aparición de pudriciones y necrosis en los tejidos de los tubérculos.

\section{Análisis proximal}

Para la caracterización química de los tubérculos se realizó el análisis proximal de las muestras tratadas que presentaron los mejores resultados de calidad postcosecha y una muestra fresca (cosechada dos días antes). Se tomaron como referencia los requisitos establecidos en las Normas AOAC, ICC y FAO para el contenido de: humedad (AOAC 925.10), cenizas (AOAC 923.03), proteína (AOAC 2001.11), extracto etéreo (AOAC 934.01), fibra cruda (ICC 113), carbohidratos totales (FAO) y valor calórico (FAO); los análisis fueron realizados por triplicado [9], con muestras de $100 \mathrm{~g}$. El análisis proximal fue realizado en el Departamento de Ciencia de Alimentos y Biotecnología (DECAB) de la Escuela Politécnica Nacional.

\section{Análisis sensorial}

Se usó una prueba discriminativa de comparación múltiple para determinar la preferencia entre los tubérculos correspondientes al mejor tratamiento físico (irradiación), el mejor tratamiento químico (aplicación de CIPC), y una muestra fresca (cosechada dos días antes). Para la cocción se utilizaron $1.5 \mathrm{~kg}$ de papa por cada tratamiento y se llevó a cabo en tres cacerolas a $91{ }^{\circ} \mathrm{C}$ durante $8 \mathrm{~min}$. En recipientes desechables codificados, se presentaron a los panelistas dos tubérculos cocidos por cada tratamiento, un vaso de agua y la respectiva hoja de evaluación. Se registró la calificación de un total de 14 panelistas de tipo juez consumidor, quienes evaluaron apariencia, color, textura y sabor [30]. Los panelistas recibieron una explicación teórica del análisis que debían realizar.

\section{Análisis estadístico}

El análisis estadístico de los resultados obtenidos se realizó con ayuda del programa Statgraphics Centurion XV.II, mediante un análisis multifactorial de varianza, y la prueba de rangos múltiples con el método de Fisher o LSD (diferencia mínima significativa), con el 95\% de confianza [31]. 


\section{RESULTADOS}

\section{Calidad postcosecha}

A continuación, se presentan los resultados obtenidos en la caracterización de las papas de la variedad chaucha amarilla tratadas con rayos gamma, con CIPC y sin tratamientos (control). En todos los casos, las papas fueron evaluadas al final de un período de almacenamiento por 40 días, a condiciones ambientales de la ciudad de Quito.

\section{Brotación externa}

En la Fig. 1 se muestran los porcentajes de brotación externa correspondientes a los tratamientos con radiación gamma y con CIPC de muestras de papa chaucha amarilla, luego de 40 días de almacenamiento. Los tratamientos que inhibieron la brotación en mayor medida fueron: la irradiación con dosis de 120 Gy y con 160 Gy y la aplicación de 30 mg CIPC kg-1 , todos a cinco días luego de la cosecha.
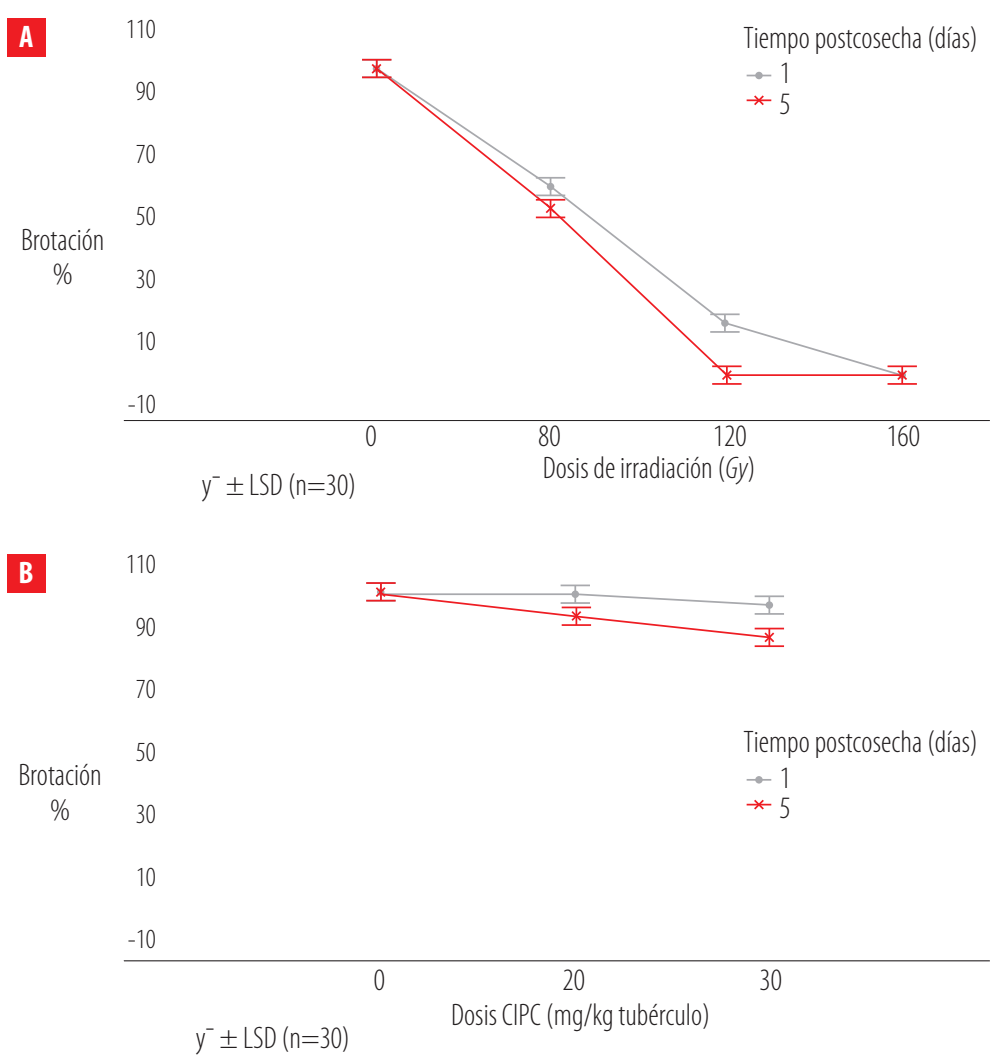

Figura 1. Porcentaje de brotación en tubérculos de Solanum phureja a) irradiados y b) tratados con (IPC, luego de 40 días de almacenamiento no refrigerado (media $\pm L S D, n=30$ ). 
En la Fig. 2 se comparan el mejor tratamiento físico y el mejor tratamiento químico para inhibir brotes con respecto al control; se encontraron diferencias significativas ( $p \leq 0.05)$ y valores de alrededor de 0\% para la irradiación a 120 Gy, 90\% para la aplicación de 30 mg CIPC kg-1 y $100 \%$ en la muestra control.

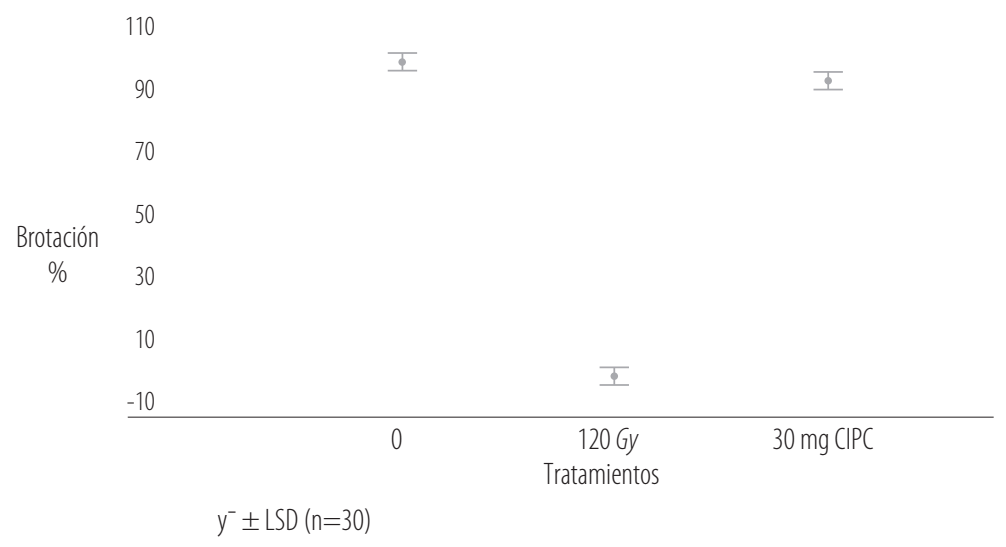

Figura 2. Porcentaje de brotación en tubérculos de Solanum phureja a) irradiados a 120 Gy y b) tratados con 30 $\mathrm{mg} \mathrm{CIPC} \mathrm{kg}{ }^{-1}$, luego de 40 días de almacenamiento no refrigerado (media \pm LSD, $n=30$ ).

\section{Diámetro y longitud de los brotes}

La muestra control presentó 100\% de brotación, con brotes de $10 \mathrm{~mm}$ de diámetro y 17 mm de longitud. En el tratamiento con irradiación a 120 Gy y 5D no se presentaron brotes y, en el caso de la aplicación de $30 \mathrm{mg} \mathrm{CIPC} \mathrm{kg}^{-1}$, se presentaron brotes de $2 \mathrm{~mm}$ de diámetro y $2 \mathrm{~mm}$ de longitud.

En la Fig. 3 se presentan imágenes de la brotación externa en los tubérculos de chaucha amarilla. Se observa una gran cantidad de brotes en la muestra control, convirtiéndola en no apta para el consumo ni la comercialización; por el contrario, las papas irradiadas presentan un aspecto similar al de las papas de pocos días de cosecha.

Los resultados del análisis estadístico y visual de la brotación se encuentran en las Fichas técnicas I y |l del material suplementario.

\section{Pérdida de peso}

En la Fig. 3 se evidencia una menor pérdida de peso en las papas irradiadas cinco días después de la cosecha (5D), con dosis de 120 y 160 Gy. También, se observó que el

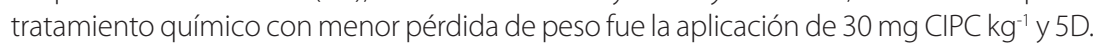

La muestra control presentó un $23.7 \%$ de pérdida de peso frente a $16.5 \%$ en el tratamiento físico con 120 Gy y $17.4 \%$ en la aplicación de $30 \mathrm{mg} \mathrm{CIPC} \mathrm{kg}{ }^{-1}$. El análisis estadístico determinó que existieron diferencias significativas $(p \leq 0.05)$ entre estos tres tratamientos, como se muestra en la Fig. 4. 


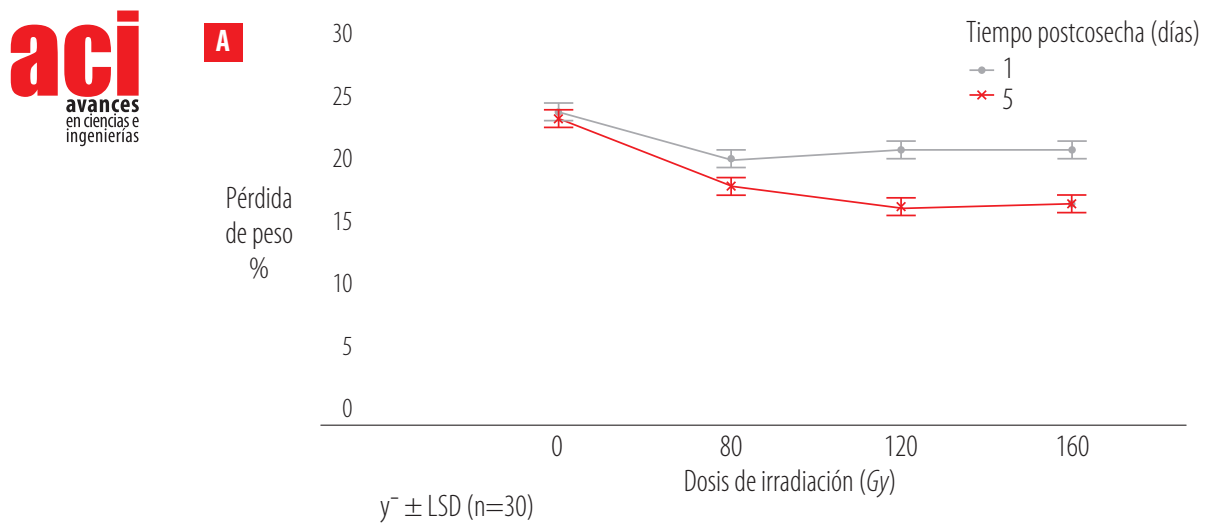

30

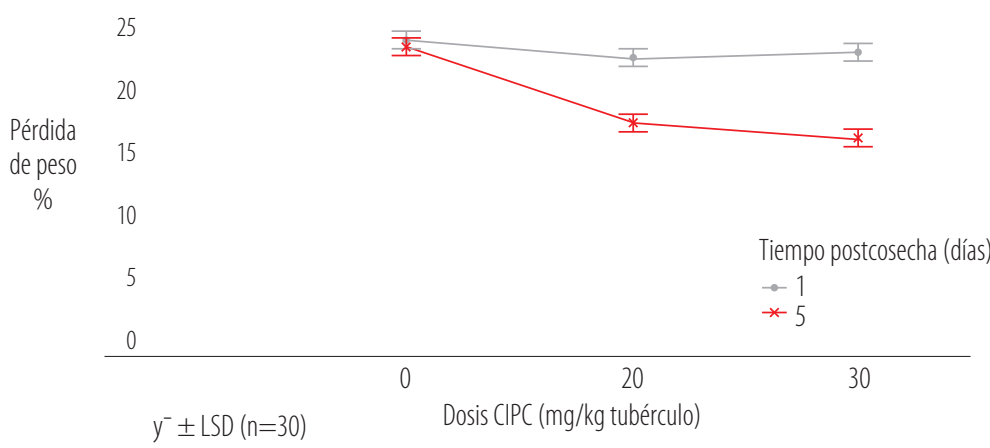

Figura 3. Porcentaje de pérdida de peso en tubérculos de Solanum phureja A) irradiados y B) tratados con (IPC, luego de 40 días de almacenamiento no refrigerado (media $\pm L S D, n=30$ ).

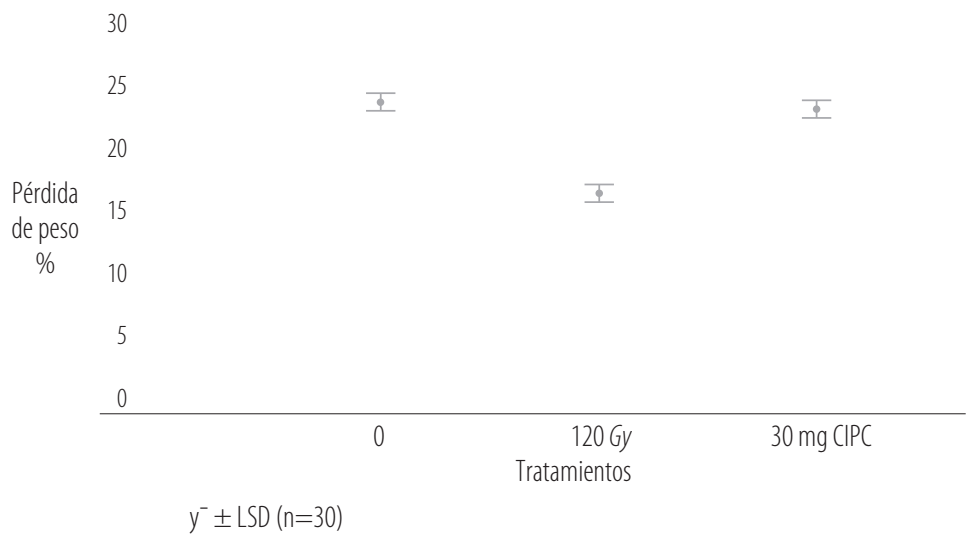

Figura 4. Porcentaje de pérdida peso en tubérculos de Solanum phureja a) irradiados a 120 Gy y b) tratados con $30 \mathrm{mg} \mathrm{CIPC} / \mathrm{kg}$, luego de 40 días de almacenamiento no refrigerado (media $\pm L S D, n=30$ ). 
Los resultados detallados del análisis estadístico de la pérdida de peso se encuentran en la Ficha técnica III del material suplementario.

\section{Pudrición}

En la Fig. 5 se observan los porcentajes de pudrición en la papa correspondiente a cada tratamiento. Se evidencia que, tanto a mayor dosis de irradiación como a mayor concentración de CIPC, existió un aumento en el porcentaje de pudrición. Sin embargo, la pudrición en las papas irradiadas con 120 Gy y 5D fue significativamente menor ( $p$ $\leq 0.05$ ) frente a papas tratadas con $30 \mathrm{mg} \mathrm{CIPC} \mathrm{kg}^{-1}$ y 5D. En la Fig. 6 se observa que la pudrición promedio fue de un 4\% al aplicar el tratamiento con la dosis de 120 Gy, 8\% al aplicar CIPC y $7 \%$ en las muestras control.

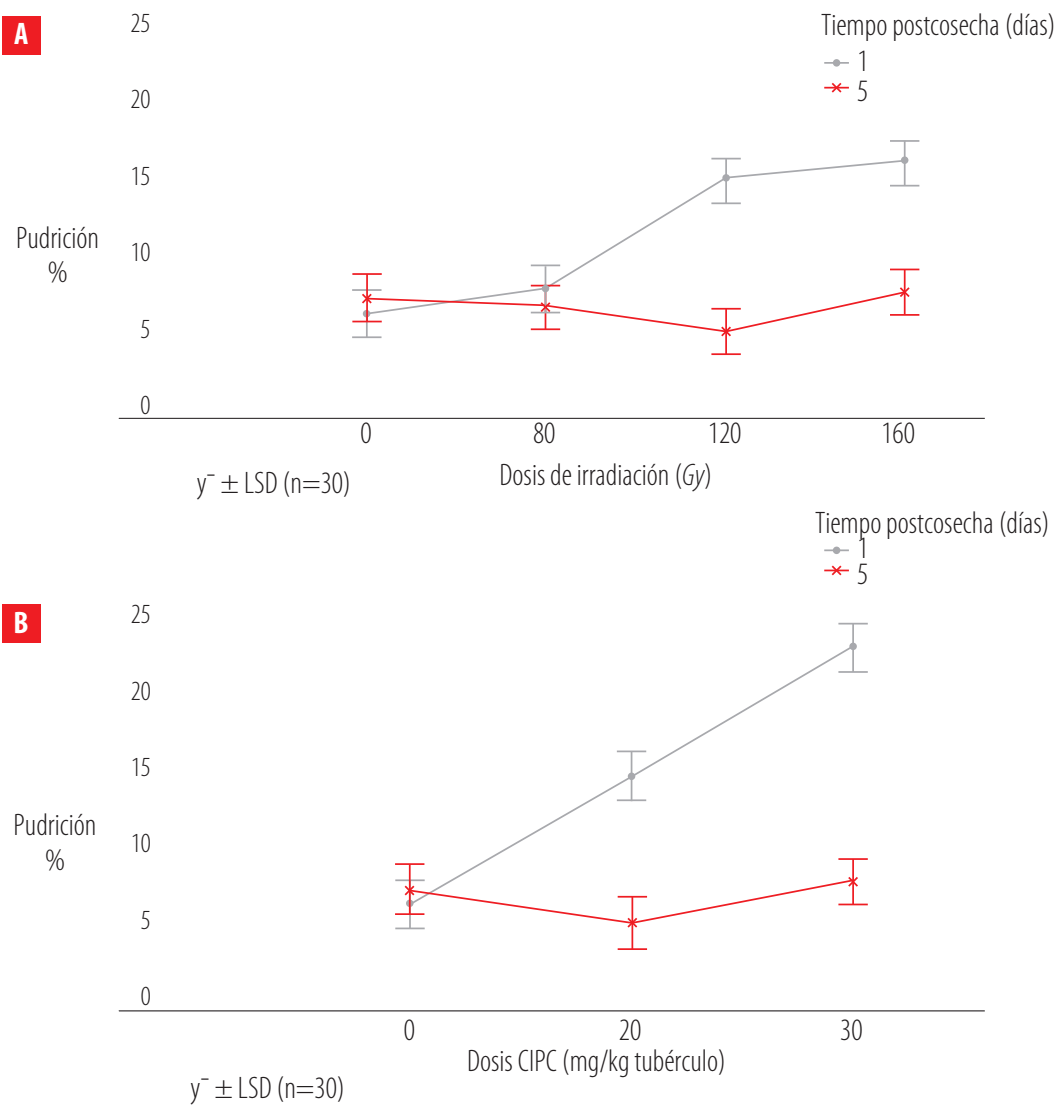

Figura 5. Porcentaje de pudrición en tubérculos de Solanum phureja A) irradiados y B) tratados con (IPC, luego de 40 días de almacenamiento no refrigerado (media \pm LSD, $n=30$ ). 


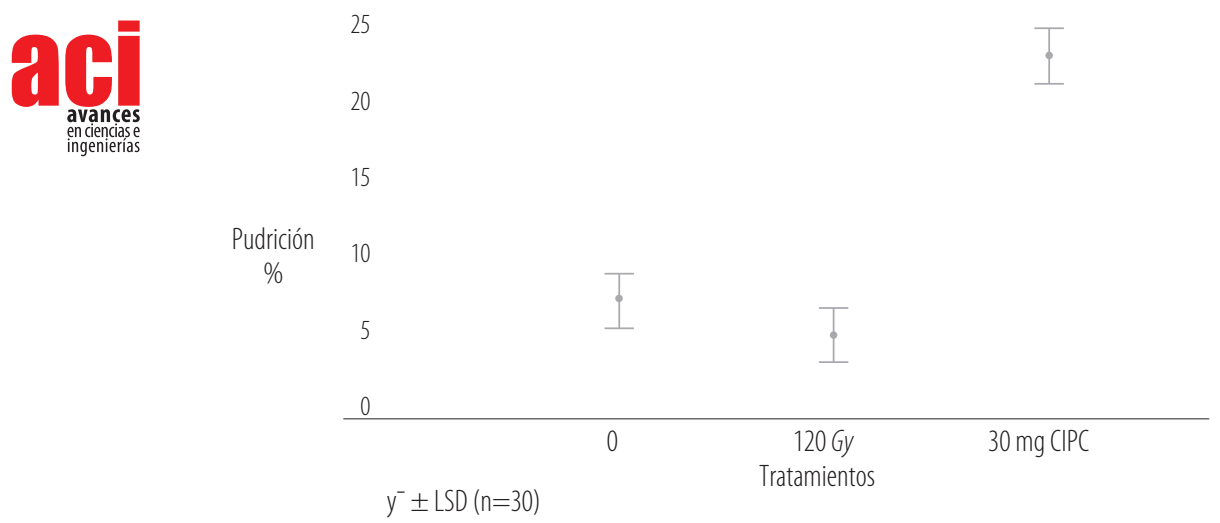

Figura 6. Porcentaje de pudrición en tubérculos de Solanum phureja a) irradiados a 120 Gy y b) tratados con 30 $\mathrm{mg} \mathrm{CIPC} \mathrm{kg}{ }^{-1}$, luego de 40 días de almacenamiento no refrigerado (media \pm LSD, $n=30$ ).

Los análisis estadístico y visual de la pudrición pueden encontrarse en las Fichas técnicas IV y $\vee$ del material suplementario.

\section{Pérdida de firmeza}

En cuanto a la firmeza, también se observaron diferencias significativas $(p \leq 0.05)$ entre los tratamientos. Se presentó una pérdida de firmeza del 20.9\% para la muestra control, frente a $17.2 \%$ en el tratamiento con la dosis de 120 Gy y de $21 \%$ en el tratamiento con $30 \mathrm{mg} \mathrm{CIPC} \mathrm{kg}^{-1}$ y 5D. Los detalles del análisis estadístico referente a la firmeza se encuentran en la Ficha técnica VI del material suplementario.

\section{Apariencia general y tiempo de vida útil}

La muestra control presentó un tiempo de vida de útil de siete días y fue no apta para el consumo a los 40 días de almacenamiento por la presencia de brotes. El mejor tratamiento

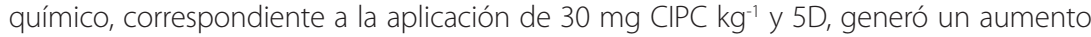
en el tiempo de vida útil de 18 días. El mejor tratamiento físico, de 120 Gy y 5D, permitió incrementar la vida útil de la papa chaucha amarilla en aproximadamente 33 días.

Los detalles del análisis estadístico de la apariencia general y el tiempo de vida útil de los tubérculos se encuentran en las Fichas técnica VII y VIII del material suplementario.

\section{Análisis proximal}

Los resultados del análisis proximal de las muestras tratadas y la muestra control fresca se presentan en la Fig. 7. Se observa que no existieron diferencias significativas ( $p>0.05)$ entre las papas del mejor tratamiento físico 120 Gy y 5D. El mejor tratamiento químico 30 mg CIPC kg-1 y 5D y la muestra control fresca en cuanto al contenido de cenizas, proteína, extracto etéreo y fibra cruda. La humedad disminuyó en aproximadamente un $10 \%$ en las muestras tratadas, que fueron almacenadas por 40 días, mientras que el porcentaje de carbohidratos totales se incrementó también en un 10\%. 


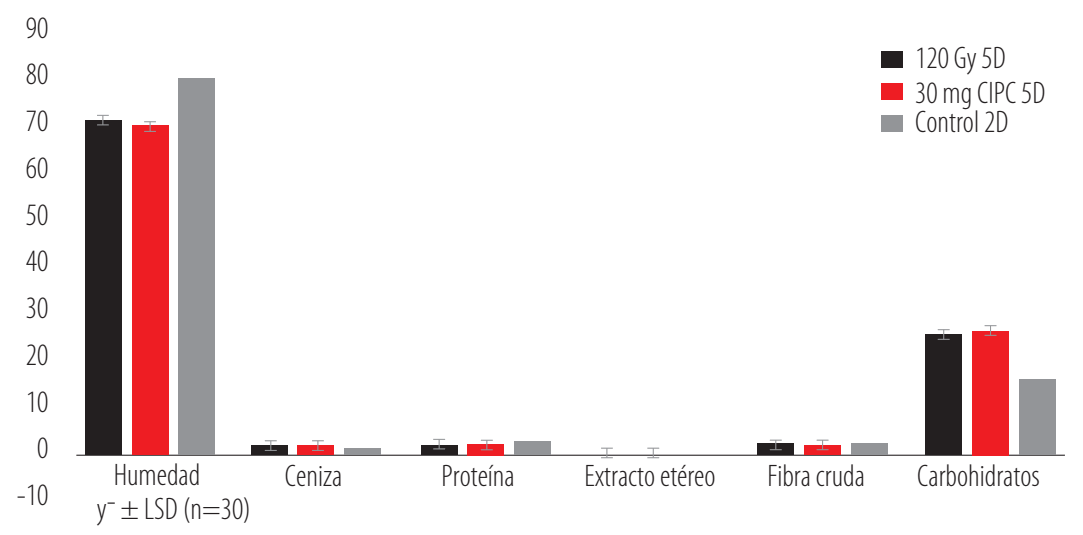

Figura 7. Análisis proximal (\% g/100 g) en tubérculos cocidos de Solanum phureja irradiada a $120 \mathrm{~Gy}$, tratada con $30 \mathrm{mg} \mathrm{CIPC} \mathrm{kg-1y} \mathrm{muestra} \mathrm{fresca} \mathrm{(2D).}$

En la Ficha IX del material suplementario están colocados los resultados de los análisis proximales realizados.

\section{Análisis sensorial}

Los resultados del análisis sensorial realizado por un grupo de 14 panelistas se muestran en la Fig. 8, donde se observa que no existieron diferencias significativas ( $p>0.05)$ entre las muestras de papa chaucha amarilla que corresponden al mejor tratamiento físico de conservación y la muestra control fresca, en los parámetros color de la pulpa y textura. En los parámetros apariencia y sabor, las muestras irradiadas tuvieron una mejor calificación que las muestras frescas. En la Ficha técnica IX del material suplementario se pueden observar las hojas entregadas a los panelistas.

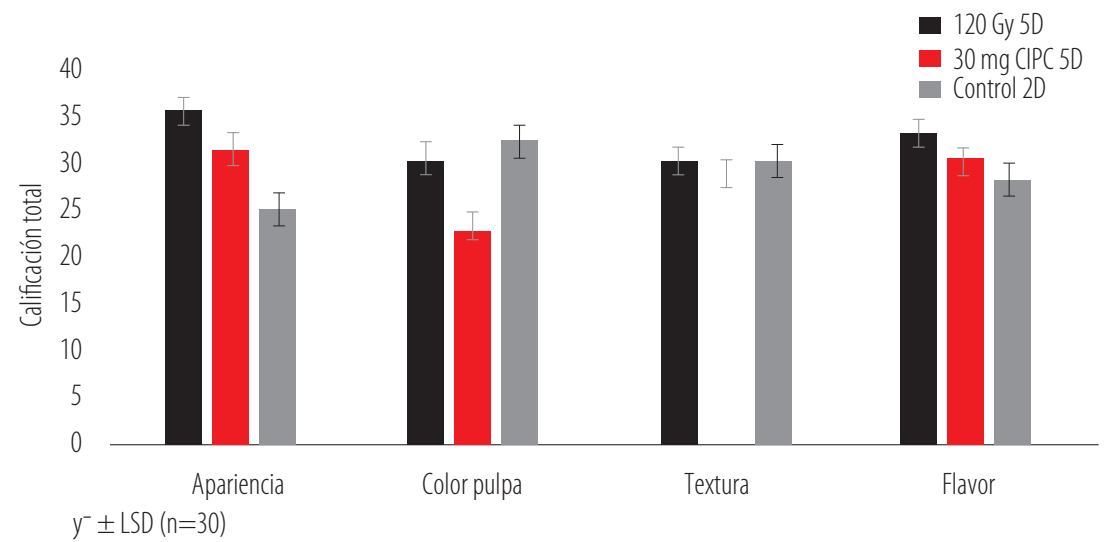

Figura 8. Análisis sensorial en tubérculos cocidos de Solanum phureja, irradiada a $120 \mathrm{~Gy}$, tratada con $30 \mathrm{mg}$ CIPC kg-1 y muestra fresca (2D). 


\section{DISCUSIÓN \\ Brotación externa}

El mejor tratamiento fue la irradiación gamma con dosis de 120 Gy, debido a la ausencia de brotes. Con la dosis de 160 Gy se obtuvo un similar efecto de inhibición, pero se eligió el primero porque una menor dosis tendría un menor costo; además, hubo ausencia de necrosis en los tubérculos tratados con la dosis de 120 Gy.

\section{Diámetro y longitud de los brotes}

La inhibición de brotes mediante irradiación se debe principalmente a los efectos sobre los tejidos meristemáticos; en primera instancia, se interrumpe la síntesis de ATP y por ello no se completa el proceso de respiración celular, esto provoca una disminución en la tasa de síntesis del ADN y ARN y el consecuente bloqueo de la división celular [32, 33].

El crecimiento de los brotes perjudica de forma directa a la calidad de la papa, además, provoca una pérdida de peso y de turgencia, una disminución de la firmeza y afecta sus propiedades nutricionales por la hidrólisis del almidón y de las proteínas, la acumulación de azúcares simples, y la disminución del contenido de vitamina $C[12,34]$. Por lo tanto, la inhibición de brotes es un factor clave para el adecuado almacenamiento y la industrialización de papas. En el caso específico de la papa chaucha amarilla, se consideran tubérculos germinados a aquellos en los que los brotes miden $2 \mathrm{~mm}$ o más [5]. La Norma INEN 1516 acepta papas con brotes de hasta $3 \mathrm{~mm}$ de longitud para el consumo humano, mientras que dentro del comercio intereuropeo no se admiten brotes [27, 35].

\section{Pérdida de peso}

Todos los tubérculos perdieron peso durante el almacenamiento, lo cual se explica principalmente porque fueron almacenados a condiciones ambientales de la ciudad de Quito (en promedio $19^{\circ} \mathrm{C}$ y $60 \% \mathrm{HR}$ ). La temperatura y humedad relativa óptimas para el almacenamiento a largo plazo de papas pueden cambiar en función de la variedad, pero se encuentran entre 8 y $12{ }^{\circ} \mathrm{C}$, y entre 85 y $90 \%$, respectivamente [12]. A temperaturas mayores o humedades relativas menores se incrementan la tasa de respiración y la brotación, lo que a su vez genera un aumento de la pérdida de peso.

La menor pérdida de peso en las muestras irradiadas se explica por la radiólisis del agua, que genera especies químicas muy reactivas $\left(\mathrm{H}^{\cdot} \cdot \mathrm{OH}, \mathrm{H}_{2} \mathrm{O}_{2^{\prime}} \mathrm{H}_{2}\right.$ y $\left.\mathrm{H}_{3} \mathrm{O}^{+}\right)$, las cuales reaccionan con moléculas aledañas en tiempos muy cortos, provocando que las moléculas de agua se reordenen o queden ligadas a nuevas estructuras bioquímicas [36]; como consecuencia, se inhabilita la síntesis de la enzima ácido indol acético (AIA) por activación de una proteasa que acelera su degradación [32], lo cual a su vez inhibe la respiración y transpiración celular, de modo que existe un menor consumo de glúcidos y, por ende, una mínima pérdida de peso [12].

Thomas y Sparks [32] concluyeron que aplicar dosis entre 50 y 200 Gy provocan una reducción de más del $50 \%$ en la pérdida de peso en todas las variedades de papa irradiadas, en comparación con sus respectivos controles. 
Disminuir la pérdida de peso es de suma importancia, ya que se trata de un parámetro de calidad para la comercialización, por ejemplo, en las papas destinadas al consumo humano y a la industrialización dentro del comercio intereuropeo se tolera entre el 2 y $6 \%$ de pérdida de peso en los tubérculos [35].

\section{Pudrición}

Para la selección de los tubérculos, se consideró la normativa ecuatoriana (Norma NTN INEN 1516), que admite un 10\% de los tubérculos con no más del 1\% de pudriciones húmeda y parda [27]. Esta decisión tuvo el propósito de verificar si el tratamiento propuesto funcionaría con una muestra de papas similar al producto que se comercializa en los mercados locales.

Algunos autores manifiestan que la necrosis y la pudrición debidas a la irradiación de tubérculos se producen por daños en el ADN superiores al 3\%, que disminuyen la síntesis del ácido nucleico [18, 32]. En el experimento se pudo constatar que dosis de 160 Gy provocaron la necrosis de los tubérculos en un 17\%, lo cual muestra que dosis por encima del valor óptimo sobre papa chaucha amarilla provocan efectos indeseables.

Al considerarse entre los principales factores de pérdida postcosecha durante el almacenamiento y la comercialización, es de suma importancia mantener un bajo porcentaje de pudrición o necrosis. El comercio intereuropeo acepta como máximo el 3\% (en peso y no más de $3.5 \mathrm{~mm}$ de profundidad) de pudrición en tubérculos para consumo humano [35], la Norma INEN 1516 tolera hasta 5\% de daños fisiológicos para papa de consumo [27].

\section{Pérdida de firmeza}

La pérdida de firmeza en papas se debe a factores ambientales y al porcentaje de germinación de los tubérculos, esto debido a que la epidermis del brote es aproximadamente 100 veces más permeable al agua en comparación con el resto del tubérculo [12].

La pérdida de firmeza en tubérculos irradiados se debe a una disminución de hasta un $50 \%$ de glúcidos como el almidón, la celulosa y la pectina $[33,36]$. En papa irradiada se ha reportado la degradación o debilitamiento de los gránulos de la membrana y la despolimerización o acortamiento de las cadenas de amilosa y/o amilopectina, debido a la ruptura de los enlaces glicosídicos [37].

En el presente estudio, las menores pérdidas de firmeza se presentaron en muestras irradiadas a 120 y 160 Gy, asociadas con una baja tasa de respiración, la no existencia de brotes y una menor pérdida de peso. Resulta clave mantener la firmeza de los tubérculos ya que repercute en su comercialización; así, el comercio intereuropeo no admite tubérculos marchitos y contraídos que han perdido su firmeza [35], mientras que la Norma INEN 1516 manifiesta como requisito tubérculos firmes y de aspecto fresco para papa de consumo [27]. 


\section{Apariencia general y tiempo de vida útil}

Los tubérculos tratados con $30 \mathrm{mg} \mathrm{CIPC} \mathrm{kg}^{-1}$ (5D) y aquellos expuestos a la mejor dosis de irradiación aumentaron su tiempo de vida útil, ya que mantuvieron por un mayor número de días su apariencia y fueron aptos para el consumo, con base en las características descritas en la Norma INEN 1516, que establece: "tubérculos limpios y firmes, libres de defectos fisiológicos y mecánicos, sin germinar o con brotes inferiores a $3 \mathrm{~mm}$ de longitud, se acepta hasta el 5\% (en peso) en daños mecánicos y defectos fisiológicos" [27]. Para el comercio intereuropeo se establecen requisitos similares, pero la aceptación en daños mecánicos y defectos fisiológicos es del 3\% en peso [35].

En un estudio similar en papa [23] se concluyó que la irradiación con dosis entre 0.08 y 0.12 kGy es una técnica postcosecha eficaz para conservar la apariencia y extender la vida útil de papas "Kufri Sindhuri", almacenadas a $22^{\circ} \mathrm{C}$ durante 120 días.

\section{Análisis proximal}

Los resultados permiten inferir que las características nutricionales de la papa chaucha amarilla se conservaron en ambos tratamientos durante el almacenamiento [38]. El incremento en el porcentaje de carbohidratos totales se debe a la pérdida de humedad, ya que dicho parámetro fue calculado por la diferencia del 100\% y los porcentajes de los otros componentes.

En la actualidad, los consumidores buscan alimentos que beneficien su dieta y se tengan beneficios para la salud; el aporte nutricional de esta variedad de papa está representado por su alto contenido de carbohidratos (principalmente almidón), alto contenido de proteína (si se compara con otros tubérculos), bajo contenido de grasa y fitoquímicos bioactivos $[9,39]$.

La tecnología de irradiación gamma permitió conservar las características nutricionales en los tubérculos tratados por un tiempo más prolongado, en comparación con los tubérculos no irradiados [23, 39].

\section{Análisis sensorial}

Existió una buena aceptación de la papa chaucha amarilla irradiada por parte de los panelistas; algunas personas manifestaron que observaron un color amarillo más intenso en la pulpa y un sabor dulce agradable al momento de la degustación en ambas muestras tratadas (física o químicamente), lo cual indica que las características organolépticas no se vieron perjudicadas en ninguno de los dos casos [38].

La aceptabilidad por parte de los consumidores para productos irradiados es muy importante [39]. Un estudio similar al presente, llevado a cabo en Chile, evaluó la aceptación pública de tubérculos irradiados; se irradiaron 164 variedades de papa a 100 Gy y se almacenaron por ocho meses (a 6-10 ${ }^{\circ} \mathrm{C}$ y $80-85 \%$ HR). No se observaron cambios en las cualidades organolépticas o culinarias al momento de la cocción y los análisis sensoriales, en los que participaron un total de 4000 personas, revelaron una ligera preferencia por papas irradiadas en comparación con las muestras no irradiadas [23,32]. La industria alimentaria ha realizado ensayos de comercialización de tubérculos 
irradiados en Hungría, Italia, Sudáfrica y Uruguay y los resultados revelan una aceptación positiva por parte de los consumidores al degustar papas irradiadas [32].

\section{CONCLUSIONES}

La muestra control (sin tratamientos) presentó $23.7 \%$ de pérdida de peso, pérdida de firmeza de 20.9\%, 7.0\% de pudrición y aparecimiento de brotes a los seis días de almacenamiento a $19{ }^{\circ} \mathrm{C}$ y $60 \%$ HR. El mejor tratamiento físico correspondió a la irradiación con una dosis de 120 Gy, cinco días después de la cosecha. Con este tratamiento se obtuvo una pérdida de peso de 16.5\%, pérdida de firmeza de $17.2 \%$, cero brotes luego de 40 días de almacenamiento; existió un 4\% de pudrición y 3\% de necrosis al final del período. El mejor tratamiento químico fue con $30 \mathrm{mg}$ de CIPC kg-1 de tubérculos, cinco días después de la cosecha. Los mismos parámetros de calidad obtuvieron valores de $16.4 \%$ a 21\% y aparecimiento de brotes a los 25 días. Existió un 8\% de pudrición y $5 \%$ de necrosis al final del período.

De acuerdo con el análisis proximal y el análisis sensorial realizado en papas cocidas, la papa chaucha amarilla irradiada con la mejor dosis tuvo una buena aceptación y sus propiedades organolépticas y nutricionales no resultaron perjudicadas con respecto a las muestras frescas, salvo por una pérdida de humedad y la aparición de un sabor dulce agradable que estarían relacionados con el tiempo de almacenamiento.

Estos resultados sugieren que la irradiación gamma de papa chaucha amarilla con la dosis adecuada permite incrementar su vida útil en aproximadamente 33 días. Esto resultaría de gran beneficio para los productores, industrializadores y consumidores.

\section{AGRADECIMIENTOS}

Los autores agradecen a los Departamentos de Ciencias Nucleares y de Ciencia de Alimentos y Biotecnología de la Escuela Politécnica Nacional por su apoyo para la investigación realizada.

\section{CONTRIBUCIONES DE LOS AUTORES}

Eddy Anguisaca llevó a cabo la parte experimental y construyó las tablas y gráficos de resultados; María Cuesta contribuyó con la revisión bibliográfica y la discusión de resultados; Marco Sinche fue el director del proyecto, supervisó el análisis estadístico y corrigió el manuscrito. 


\section{REFERENCIAS}

[1] Centro Internacional de la Papa. (2015). Dato y cifras de la papa - International Potato Center. Recuperado de https:// web.archive.org/web/20180906073207/https://cipotato.org/es/lapapa/dato-y-cifras-de-la-papa/Consultado el 27 de septiembre de 2020.

[2] Asociación Latinoamericana de la Papa. (2014). XXVI Congreso ALAP 2014 - Bogotá, Colombia. Recuperado de http:// www.papaslatinas.org/alap2014/ Consultado el 29 de septiembre de 2020.

[3] Pumisacho, M. y Sherwood, S. (2002). El cultivo de la papa en el Ecuador. Recuperado de https://web.archive.org/ web/20201031010112/https://cipotato.org/wp-content/uploads/Documentacion PDF/Pumisacho y Sherwood Cultivo de Papa en Ecuador.pdf Consultado el 30 de octubrebre de 2020.

[4] Mancero, L. (2012). Estudio de la Cadena de la Papa en Ecuador FAO-ESA / CIP. Recuperado de: https://web.archive.org/ web/20150501104951/http://www.fao.org/fileadmin/templates/esa/LISFAME/Documents/Ecuador/cadena_papa. pdf Consultado el 30 de octubrebre de 2020.

[5] Chalapud, M. A., Caicedo, C., Ruiz, E. M., \& Valencia, M. F. (2016). Preservation properties: Chitosan-based coatings and aloe vera applied in creole potato (Solanum phureja). Informador Técnico, 80(1), 9-19.

[6] Rodríguez, D. R., \& Ramírez, L. N. (2011). La agroindustria de la papa criolla en Colombia. Situación actual y retos para su desarrollo. Gestión y Sociedad, 4(2), 17-30.

[7] Solorzano, V., Rincón, A., Villacrés, E. y Arguello, P. F. (2015). Recubrimiento comestible para látex de sande (Brosimiun utile) como alternativa en la inhibición de brotes en papa chaucha (Solanum phureja). En P. Kromann, X. Cuesta, M. Romero, B. R. Montero y P. Cuasapaz (Eds.), VI Congreso Ecuatoriano de la Papa (pp. 1-221). Ibarra.

[8] Suárez, J. A., Cerón, M. S., Prieto, L. y Rodríguez, J. (2017). Almacenamiento de clones de papa criolla (Solanum tuberosum grupo Phureja) en atmósferas modificadas activas y pasivas . En P. Kromann, X. Cuesta, B. R. Montero, P. Cuasapaz, A. León-Reyes y A. Chulde (Eds.), Memorias del VII Congreso Ecuatoriano de la Papa. Tulcán.

[9] Peña, C. (2015). Evaluación del contenido nutricional y actividad antioxidante en Solanum tuberosum grupo Phureja. (Tesis de Maestría). Universidad Nacional de Colombia, Bogotá

[10] Piñeros, J. (2009). Recopilación de la investigación del sistema productivo papa criolla. Bogotá: Federación Colombiana de Productores de Papa. Recuperado de https://repository.agrosavia.co/handle/20.500.12324/13653 Consultado en septiembre de 2020.

[11] Toro, M. (2016). Evaluación del efecto de 3 inhibidores de brotación en papa criolla (Solanum Phureja) variedad criolla Colombia aplicados en el proceso de poscosecha (Tesis de Doctorado). Universidad Nacional de Colombia , Bogotá.

[12] Paul, V., Ezekiel, R., \& Pandey, R. (2016). Sprout suppression on potato: need to look beyond CIPC for more effective and safer alternatives. Journal of Food Science and Technology, 53(1), 1-18. Doi: http://doi.org/10.1007/s13197-015-1980-3

[13] Bradshaw, N. (2006). Pesticide residue minimisation-potatoes (N. Bradshaw \& S. Ogilvy, Eds.). UK: Food Standards Agency.

[14] Aliaga, I., Velásquez, F., Mendoza, R., \& Chuquilín, R. (2011). Efecto de la aplicación de Chlorpropham en el brotamiento de tubérculos de olluco (Ullucus tuberosus L.) en condiciones de almacén. Scientia Agropecuaria, 2(2), 91-96.

[15] Frazier, M. J., \& Olsen, N. L. (2015). The Effects of Chlorpropham Exposure on Field-Grown Potatoes. American Journal of Potato Research, 92(1), 32-37. Doi: http://doi.org/10.1007/s12230-014-9408-6

[16] Calleja, A., Lanzarote, I., Björkroth, J., González, R., Moragrega, R., Alonso, J., Cocolin, L., Mate, A., Clemente, R., Estaca, J., \& López, B. (2010). Nuevas tecnologías en la conservación y transformación de los alimentos (Vol. 178). Madrid: International Marketing and Communication.

[17] International Atomic Energy Agency. (2017). Manual de buenas prácticas para la irradiación de alimentos: Aplicaciones sanitarias, fitosanitarias y de otro tipo (Núm. 481). Viena.

[18] Maraver, J., Moreno, I., Jos, A., \& Cameán, A. (2012). Irradiación de alimentos: Toxicología alimentaria. Madrid: Díaz de Santos.

[19] Food and Drug Administration. (2016). Hechos sobre alimentos: La irradiación de alimentos. Recuperado de https://web. archive.org/web/20201001173346/https://www.fda.gov/media/81266/download Consultado el 28 de septiembre de 2020. 
[20] Eustice, R. (2016). Novedades sobre Irradiación de Alimentos del 2016. Recuperado de http://www.cafi.org.ar Consultado el 29 de septiembre de 2020.

[21] International Atomic Energy Agency. (2015). Manual of good practice in food irradiation: Sanitary, phytosanitary and other applications. Vienna.

[22] Nordion. (2011). The History of Food Irradiation. Recuperado de https://web.archive.org/web/20201001173003/ http://www.nordion.com/wp-content/uploads/2014/10/GT_History-of-Food-Irradiation.pdf Consultado el 29 de septiembre de 2020.

[23] Mahto, R., \& Das, M. (2014). Effect of gamma irradiation on the physico-mechanical and chemical properties of potato (Solanum tuberosum L.), cv. 'Kufri Sindhuri', in non-refrigerated storage conditions. Postharvest Biology and Technology, 92(1), 37-45. Doi: http://doi.org/10.1016/j.postharvbio.2014.01.011

[24] Soares, I. G. M., da Silva, E. B., Amaral, A. J., Machado, E. C. L., \& Silva, J. M. (2016). Physico-chemical and sensory evaluation of potato (Solanum tuberosum L.) after irradiation. Anais da Academia Brasileira de Ciencias, 88(2), 941950. Doi: http://doi.org/10.1590/0001-3765201620140617

[25] Zabala, D. (2011). Efecto de diferentes dosis de radiación gamma sobre la expresión fenotípica en papa criolla (Solanum tuberosum grupo phureja, variedad criolla Colombia) (Tesis de Licenciatura). Universidad Distrital Francisco José de Caldas, Bogotá.

[26] Guzmán, J. D. (2016). Caracterización fenotipica de un cultivo de papa criolla (Solanum Tuberosum Grupo Phureja, Variedad (riolla Colombia) irradiada con Cobalto 60 ubicado en el Municipio El Rosal, Finca el Pino Km 16 Vía Subachoque Cundinamarca (Tesis de Licenciatura). Universidad Distrital Francisco José de Caldas, Bogotá.

[27] Servicio Ecuatoriano De Normalización. (2012). Hortalizas frescas. Papas. Requisitos. Recuperado de https://web. archive.org/web/20201001172851/https://www.normalizacion.gob.ec/buzon/normas/1516.pdf Consultado el 28 de septiembre de 2020.

[28] Suttle, J. C. (2007). Dormancy and sprouting. En D. Vreugdenhil, J. Bradshaw, C. Gebhardt, F. Govers, M. A. Taylor, D. K. L. MacKerron y H. A. Ross (Eds.), Potato biology and biotechnology (1a ed., pp. 287-309). Ámsterdam: Elsevier Science BV.

[29] Ruiz, E. (2016). Estudio del efecto del tratamiento poscosecha por irradiación gamma sobre la vida útil de la cebolla perla ecuatoriana de exportación (Allium cepa L.) (Tesis de Ingeniería). Escuela Politécnica Nacional. Quito.

[30] Díaz Herrera, M. Á., \& Gutiérrez Guzmán, N. (2013). Evaluación sensorial de papa criolla (Solanum phureja) congelada criogénicamente. Ingeniería y Región, 10, 143. Doi: http://doi.org/10.25054/22161325.766

[31] Gutiérrez, H., \& De la Vara, R. (2008). Análisis y diseño de experimentos. México, D.F: Mc Graw Hill.

[32] Thomas, P., \& Sparks, W. (2009). Radiation preservation of foods of plant origin: Part 1. potatoes and other tuber crops. Critical Reviews in Food Science and Nutrition, 19(4), 327-379. Doi: http://doi.org/10.1080/10408398409527380

[33] Urbain, W. M. (1986). Radiation chemistry of food components and of foods. En Food Irradiation (pp. 37-82). Academic Press, Inc. Doi: http://doi.org/10.1002/9781118422557.ch6

[34] de Freitas, S. T., Pereira, E. I. P., Gomez, A. C. S., Brackmann, A., Nicoloso, F., \& Bisognin, D. A. (2012). Processing quality of potato tubers produced during autumn and spring and stored at different temperatures. Horticultura Brasileira, 30(1), 91-98. Doi: http://doi.org/10.1590/S0102-05362012000100016

[35] Comité Europeo de RUCIP. (2017). Reglas y Usos del Comercio Intereuropeo de la Patata. Recuperado de https://web. archive.org/web/20201001172218/http://europatat.eu/wp-content/uploads/2016/10/RUCIP-2017-ES.pdf

[36] Suárez, R. (2001). Conservación de alimentos por radiación. Redalyc, 4(6), 85-124. Recuperado de https://web. archive.org/web/20201001171502/https://www.redalyc.org/pdf/877/87740608.pdf Consultado en septiembre 2020.

[37] Atrous, H., Benbettaieb, N., Chouaibi, M., Attia, H., \& Ghorbel, D. (2017). Changes in wheat and potato starches induced by gamma irradiation: A comparative macro and microscopic study. International Journal of Food Properties, 20(7), 1532-1546. Doi: http://doi.org/10.1080/10942912.2016.1213740

[38] Rossi, L., Watson, D., Escandarani, S., Miranda, A., \& Troncoso, A. (2009). La radiación a la mesa. Revista chilena de infectología, 26(4), 318-330. Doi: http://doi.org/10.4067/S0716-10182009000500003

[39] Furrer, A. N., Chegeni, M., \& Ferruzzi, M. G. (2018). Impact of potato processing on nutrients, phytochemicals, and human health. Critical Reviews in Food Science and Nutrition, 58(1), 146-168. Doi: http://doi.org/10.1080/1040839 8.2016.1139542 